\title{
A Common Basis for Analytical Clutter Representations
}

\author{
John E. Gray, Naval Surface Warfare Center Dahlgren Division
}

\author{
Keywords: Analytical Clutter Model, Probability Density \\ Function, Phase Noise
}

\section{SUMMARY \& CONCLUSIONS}

In order to understand the problems intractable problems nature presents us, we are forced to make simplifications till we arrive at simple analytical models that we are capable of understanding. Such canonical models are rare, but useful as tools for constructing more realistic models that we can use analyze nature. The class of analytical models for clutter analysis limited to those that consist of various amplitude models with the phase noise assumed to have a probability density function that is uniformly distributed. These analytical models can be extended by relaxing the assumption of uniform phase noise to phase noise with non-uniform distributions. It is shown how to determine the probability density function for these non-uniform distributions in general and then the method is illustrated with such distributions as Gaussian, Laplacian, and Chi-squared. This enables one to determine the probability density functions of the individual components of clutter model such as $\hat{x}_{t}=\hat{v}_{t} \cos \left(\hat{\phi}_{c}\right)$.

Using the rule for determining the products of distributions, we show that the functional form for $\hat{x}_{t}$ is reduced to evaluating integrals that reduce to elliptical functions. Once the functional form has been determined, it is easy to determine the moments of the PDF and hence completely characterize its statistics.

\section{INTRODUCTION}

Analytical clutter models have proven useful in both the design and analysis of moving target indicators (MTI) and pulse Doppler (PD) radars. Usage of them to represent random clutter enables one to predict some aspects of system performance analytically, which is always useful as means of testing simulations and to provide sanity checks for ones data [1], [2]. It is not generally realized that most clutter models can be cast in the form

$$
\hat{c}_{t}=\hat{v}_{t} \cos \left(\omega_{c} t+\hat{\phi}_{c}\right)
$$

where "hat" denotes random variable, $\hat{c}_{t} \stackrel{\circ}{=}$ distributed clutter process that is assumed to be strict sense stationary, $\hat{v}_{t} \stackrel{\circ}{=}$ clutter envelope process, $\omega_{c} \stackrel{\circ}{=}$ clutter frequency, $\hat{\phi}_{c} \stackrel{\circ}{=}$ phase noise process (which we assume is not uniform). (I will refer to this as the communication model throughout the paper.) If we define

$$
\hat{x}_{t}=\hat{v}_{t} \cos \left(\hat{\phi}_{c}\right),
$$

and

$$
\hat{y}_{t}=\hat{v}_{t} \sin \left(\hat{\phi}_{c}\right),
$$

the clutter process can also be represented as

$$
\hat{c}_{t}=\hat{x}_{t} \cos \left(\omega_{c} t\right)-\hat{y}_{t} \sin \left(\omega_{c} t\right) .
$$

If we take $\hat{v}_{t}$ to be a zero mean process with a variance $\sigma^{2}$, and assume the phase noise is not a random process, then this is the standard Gaussian process with the clutter being independent of the time origin. Thus the Gaussian clutter process is a special case of the communication model. Similarly the Rician Clutter model is just the Gaussian model with a scatter $(S)$ added to the mixture. The log-normal clutter model is to take the communication model for clutter with the envelope probability distribution taken to Rayleigh distributed and the phase noise taken to be uniformly distributed. Other models of the amplitude that have been used include the Weibull representation and the K-distribution. The problem with these approaches to analytical clutter is they treat the amplitude differently, but the not the phase. All analytical models assume that the phase noise is uniformly distributed. This is clearly not the case, but there has been no method that overcomes this difficulty. In this paper we propose a method for characterizing non-uniform phase noise, so one can determine the phase noise clutter distribution and then use this with a desired amplitude distribution determine better analytical models for clutter. We note that the analytical clutter determination using the communication model of clutter is a special instance of the Rayleigh Problem [4], [4], [5] originally arose in Lord Rayleigh's investigations into scattering acoustical waves off of rough surfaces. The problem was to evaluate the PDF of the random sums such as

$$
\widehat{X}=\sum_{i=1}^{N} \hat{x}_{i}=\sum_{i=1}^{N} \hat{a}_{i} \cos \left(\hat{\theta}_{i}\right) .
$$

\section{DETERMINATION OF PROBABILITY DENSITY FUNCTIONS USING CHARCTERISTIC FUNCTIONS}

The communication model of clutter is the $N=1$ instance of the Rayleigh problem. In many engineering applications, difficulty occurs when we have a change of variables. We know 
the distribution of the original random variable $\hat{x}$, but we have to deal with a change of variables $\hat{y}=f(\hat{x})$, so we are trying to cope with a design based on knowledge of $\hat{x}$ rather than $\hat{y}$ which is what we actually need. A method for finding the underlying probability distribution for $\hat{y}$ is to use characteristic functions. The definition of a characteristic function $(\mathrm{CF})$ for a density $P(x)$ is

$$
M_{P}(\theta)=\left\langle e^{i \theta x}\right\rangle=\int_{-\infty}^{\infty} e^{i \theta x} P(x) d x
$$

which can also be interpreted as the expected value or average, $\left\langle e^{i \theta x}\right\rangle$, of $e^{i \theta x}$ (see [?] for a thorough discussion of CF's). The $\mathrm{CF}$ has the properties:

1) $M(0)=1$.

2) $M^{*}(-\theta)=M(\theta)$.

3) $|M(\theta)| \leq 1$.

4) $|M(\theta)| \leq M(0)$.

There are several methods for defining the Fourier transform of a function that are used in the literature; all involve the placement of the factor $2 \pi$ that always occurs when using the Fourier transform. We use the asymmetric form that is found in Bracewell, Papoulis, and others. If we adopt the Hilbert space notation for the inter-product of two functions (where $*$ denotes complex conjugate)

$$
\langle f(t), g(t)\rangle=\int_{-\infty}^{\infty} f^{*}(t) g(t) d t
$$

then the Fourier transform of a function $f(t)$ can be defined in Hilbert space notation as

$$
F(\omega)=\left\langle e^{-i \omega t}, f(t)\right\rangle=\int_{-\infty}^{\infty} e^{i \omega t} f(t) d t .
$$

The inverse Fourier transform of a function $F(\omega)$ is defined as

$$
f(t)=\frac{1}{2 \pi}\left\langle e^{i \omega t}, F(\omega)\right\rangle=\frac{1}{2 \pi} \int_{-\infty}^{\infty} e^{-i \omega t} F(\omega) d \omega .
$$

Also, note the definition of the Dirac delta function in this notation is

$$
\delta(t)=\frac{1}{2 \pi}\left\langle e^{i \omega t}, 1\right\rangle=\frac{1}{2 \pi} \int_{-\infty}^{\infty} e^{-i \omega t} d \omega .
$$

Note the definitions and results we use are consistent with the various Papoulis books with $i=-j$ as the translation device.

In many engineering applications, difficulty occurs when we have change of variables. If one has a random variable $\hat{x}$ with a density $P(x)$, one would often like to know the density of a new variable, say $\hat{u}$, that is a function of the old variable

$$
\widehat{u}=f(\widehat{x}) .
$$

The standard method for solving this type of problem is found in [7]. Assume knowledge of the characteristic function is known, then the distribution can be obtained by inversion. The characteristic function is the expected value of $e^{i \omega f(x)}$

$$
M_{u}(\omega)=\left\langle e^{i \omega f(x)}\right\rangle=\left\langle e^{-i \omega f(x)}, P_{x}(x)\right\rangle .
$$

Now if we substitute this definition of $M_{u}(\omega)$ into the definition of $P(x)$ gives

$$
P_{u}(u)=\frac{1}{2 \pi}\left\langle e^{i \omega u},\left\langle e^{i \omega f(x)}, P_{x}(x)\right\rangle\right\rangle
$$

which upon rearrangement of the order of integration gives

$$
P(u)=\frac{1}{2 \pi}\left\langle\left\langle e^{i \omega u} e^{-i \omega f(x)}, 1\right\rangle, P_{x}(x)\right\rangle
$$

follows from the definition of the delta function [8] that

$$
\delta(u-f(x))=\frac{1}{2 \pi}\left\langle e^{i \omega(u-f(x))}, 1\right\rangle
$$

SO

$$
P(u)=\left\langle\delta(u-f(x)), P_{x}(x)\right\rangle .
$$

Now one of the properties of the delta function is [9]

$$
\delta(g(x))=\sum_{i} \delta\left(x-x_{i}\right) \frac{1}{\left|g^{\prime}\left(x_{i}\right)\right|}
$$

were the $x_{i}^{\prime} s$ are the solution to $g\left(x_{i}\right)=0$, e.g. the zeros of $g$. Now for our problem, $g(x)=f(x)-u$, the derivatives of $g$ is the derivative of $f$, so we have

$$
\delta(f(x)-u)=\sum_{i} \delta\left(x-x_{i}\right) \frac{1}{\left|f^{\prime}\left(x_{i}\right)\right|} .
$$

Thus the expression for the density function becomes

$$
P(u)=\sum_{i} \frac{P\left(x_{i}\right)}{\left|f^{\prime}\left(x_{i}\right)\right|} .
$$

If $f$ is single valued, then the solution is particularly simple.

The transformation $\hat{y}=R \sin (\hat{\varphi})$ a $\operatorname{PDF} f_{\varphi}(\varphi)$ is onto but not one-to-one over the interval $[-\infty, \infty]$. Thus it has an infinite number of zeros. It is more convenient to determine the CF directly, so the Fourier transformation of the PDF is

$$
M_{\varphi}(\omega)=\left\langle e^{i \omega R \sin (\varphi)}, f_{\varphi}(\varphi)\right\rangle .
$$

The exponential can be written as

$$
\sum_{n=-\infty}^{\infty} J_{n}(\omega R) e^{j n \varphi}=e^{i \omega R \sin (\varphi)}
$$

so the CF is given by [10],[11]

$M_{\varphi}(\omega)=\sum_{n=-\infty}^{\infty} J_{n}(\omega R)\left\langle e^{i n \varphi}, f(\varphi)\right\rangle=\sum_{n=-\infty}^{\infty} J_{n}(\omega R) F(n)$;

where where $F(n)$ is the Fourier transform of the PDF for the angle variable $f_{\varphi}(\varphi)$ which is evaluated $n$. Noting the Bessel functions can be rewritten as $\left(J_{-n}(x)=(-)^{n} J_{n}(x)\right)$, we have

$$
M_{\varphi}^{\sin }(\omega)=J_{0}(\omega R) F_{s}(0)+\sum_{n=1}^{\infty} J_{n}(\omega R) S(n)
$$

where $S(n)=\left[F(n)+(-)^{n} F(-n)\right]$. If $F(n)$ is even or odd, this formula can be simplified further. For the transformation $\widehat{x}=R \cos (\hat{\varphi})=R \sin \left(\hat{\varphi}-\frac{\pi}{2}\right)$, which amounts to replacing $\varphi$ by $\varphi-\frac{\pi}{2}$ in the exponential, so the $\mathrm{CF}$ is

$$
M_{\varphi}^{\cos }(\omega)=J_{0}(\omega R) F(0)+\sum_{n=1}^{\infty} J_{n}(\omega R) C(n) .
$$


where $C(n)=\left[(-)^{n} F(n)+F(-n)\right]$. The expressions for $C(n)$ and $S(n)$ can be simplified further if the functions are even or odd.

The expression for the moments of the characteristic function can be expressed as the derivative of the $\mathrm{CF}$,

$$
\left\langle x^{m}\right\rangle=\left.\frac{1}{i^{m}} \frac{\partial^{m} M_{P}(\omega)}{\partial \omega^{m}}\right|_{\omega=0}
$$

so

$$
\left\langle x^{m}\right\rangle=\left.\frac{R^{m}}{i^{m}} 2^{m}\{S(p) / C(p)\} J_{p}^{(m)}(\omega R)\right|_{\omega=0} .
$$

To determine $\left\langle x^{n}\right\rangle$ requires us know the $\mathrm{n}$-th derivative of the Bessel function since the characteristic function for phase noise always have Bessel function components. Since $J_{0}(0)=$ 1 and $J_{n}(0)=0$ for $n \neq 0$, the only terms that remain after we take the derivative with respect to $\omega$ and set it equal to zero are those Bessel functions that have zero coefficients, e.g. those of the form $J_{p-m}(x)$ which are one when $p=m$. allows us to determine the moments to arbitrary order. Three examples of distributions are:

1) Gaussian or Normal Distribution: The PDF of the zero mean normal distribution has a $\mathrm{CF}$ given by

$$
\begin{gathered}
\frac{1}{\sqrt{2 \sigma_{\theta}^{2} \pi}} e^{-\frac{x^{2}}{2 \sigma_{\theta}^{2}}} \Leftrightarrow e^{-\sigma_{\theta}^{2} \omega^{2} / 2} \\
\text { thus } F(n)=e^{-\sigma_{\theta}^{2} n^{2} / 2}
\end{gathered}
$$

which is even. Note the mean is zero and the second moment for either transformation is

$$
\left\langle x^{2}\right\rangle=\frac{R^{2}}{2}\left[1-e^{-2 \sigma_{\theta}^{2}}\right] .
$$

The third moment is zero, and the fourth moment for either transformation is

$$
\left\langle x^{4}\right\rangle=\left.\frac{\partial^{4} M_{\varphi}(\omega)}{\partial \omega^{4}}\right|_{\omega=0}=\frac{R^{4}}{8}\left[e^{-8 \sigma_{\theta}^{2}}-4 e^{-2 \sigma_{\theta}^{2}}+3\right]
$$

For non-zero mean Gaussian, the CF is

$$
\sqrt{\frac{2}{\pi^{2} \sigma_{\theta}^{2}}} e^{-\omega^{2} \sigma_{\theta}^{2} / 2} e^{-i \omega \theta_{0}}
$$

thus

$$
F(n)=e^{-n^{2} \sigma_{\theta}^{2} / 2} e^{-i n \theta_{0},}
$$

Note the mean is

$$
\langle x\rangle=2 e^{-\sigma_{\theta}^{2} / 2}\left[\cos \theta_{0}\right] R,
$$

so the second moment is

$$
\left\langle x^{2}\right\rangle=\frac{R^{2}}{2}\left[1-e^{-2 \sigma_{\theta}^{2}} \cos \left(2 \theta_{0}\right)\right] .
$$

while the fourth moment is for either transformation

$$
\left\langle x^{4}\right\rangle=\frac{R^{4}}{8}\left[e^{-8 \sigma_{\theta}^{2}} \cos \left(4 \theta_{0}\right)-4 e^{-2 \sigma_{\theta}^{2}} \cos \left(2 \theta_{0}\right)+6\right] \text {. }
$$

2) Laplace Distribution: The PDF's CF is

$$
\frac{\alpha}{2} e^{-\alpha|x|} \Leftrightarrow \frac{\alpha^{2}}{\alpha^{2}+\omega^{2}},
$$

thus

$$
F(n)=\frac{\alpha^{2}}{\alpha^{2}+n^{2}},
$$

which is even. Note the mean is zero and the second moment is

$$
\left\langle x^{2}\right\rangle=\frac{R^{2}}{2}\left[1-\frac{\alpha^{2}}{\alpha^{2}+4}\right],
$$

while the fourth moment is

$$
\left\langle x^{4}\right\rangle=\frac{R^{4}}{8}\left[\frac{\alpha^{2}}{\alpha^{2}+16}-4 \frac{\alpha^{2}}{\alpha^{2}+4}+6\right] .
$$

3) Chi Squared $\chi^{2}(n)$ : The PDF's CF is

$$
\frac{x^{m / 2-1}}{2^{m / 2} \Gamma(m / 2)} e^{-x / 2} \Theta(x) \Leftrightarrow \frac{1}{(1-2 i \omega)^{m / 2}},
$$

where

$$
\Theta(x)=\left\{\begin{array}{ll}
1 & x \geq 0 \\
0 & x<0
\end{array},\right.
$$

thus

$$
F(n)=\frac{1}{(1-2 i n)^{m / 2}} .
$$

Note that the mean is

$$
\langle x\rangle=i\left[\frac{1}{(1+2 i)^{m / 2}}-\frac{1}{(1-2 i)^{m / 2}}\right] R
$$

and the second moment is

$$
\left\langle x^{2}\right\rangle=\left[2-\frac{1}{(1-4 j)^{m / 2}}-\frac{1}{(1+4 i)^{m / 2}}\right] R^{2} .
$$

From the characteristic function, we can determine the PDF of the transformation $\hat{y}=R \sin (\hat{\theta})$ by using a Fourier transform identity found in [12] which enables us to determine the PDF of the sinusoidal transforms from the expression for $M_{\varphi}(\omega)$ to give

$$
f_{y}^{\sin \theta}(y)=\frac{2}{\pi} \frac{\left[F(0)+\sum_{n=1}^{\infty} U_{\sin (\theta)}(n) T_{n}\left(\frac{y}{R}\right)\right]}{\sqrt{\left(1-\left(\frac{y}{R}\right)^{2}\right)}} \Theta\left(1-\left|\frac{y}{R}\right|\right)
$$

where

$$
U_{\sin (\theta)}(n)=\left[F(n)+(-)^{n} F(-n)\right](-i)^{n} .
$$

and $T_{n}(\cdot)$ are the Chebyshev polynomials. The PDF of the transformation $\hat{y}=R \cos (\hat{\theta})$ is

$$
f_{y}^{\cos \theta}(y)=\frac{2}{\pi} \frac{\left[F(0)+\sum_{n=1}^{\infty} U_{\cos (\theta)}(n) T_{n}\left(\frac{y}{R}\right)\right]}{\sqrt{\left(1-\left(\frac{y}{R}\right)^{2}\right)}} \Theta\left(1-\left|\frac{y}{R}\right|\right)
$$

where

$$
U_{\cos (\theta)}(n)=\left[(-)^{n} F(n)+F(-n)\right] .
$$

To determine the PDF from a given characteristic function, we merely substitute the particular $F(n)$ and we have the underlying PDF for sinusoidal transformations for any distribution.

\section{TWO DIMENSIONAL PDF's FOR ANALYTIC CLUTTER}


Since clutter results from scattering off a rough surface, there are four possibilities to consider for either clutter or scattering:

1) All the amplitudes are deterministic and individual random phase angles are the same type of distribution with different means and variances,

2) All the amplitudes are deterministic and individual random phase angles have different distributions,

3) All the amplitudes are random with he same type of distribution with different means and variances and individual random phase angles are the same type of distribution with different means and variances,

4) All the amplitudes are random with he same type of distribution with different means and variances and individual random phase angles are phase angles have different distributions.

Once one has the individual $\widehat{z}$, then one has to determine the PDF of $N$ random sums

$$
\widehat{Z}=\widehat{z}_{1}+\widehat{z}_{2}+\ldots+\widehat{z}_{N} .
$$

To evaluate such sums, it suffices to calculate them two-fold at a time, so sums such as $\widehat{z}_{12}=\widehat{z}_{1}+\widehat{z}_{2}$, with distributions $P_{z_{1}}\left(z_{1}\right)$ and $P_{z_{2}}\left(z_{2}\right)$ (assuming they are uncorrelated), then the distribution of $\widehat{z}$ which is denoted by $P_{z}(z)$ is

$$
\begin{aligned}
P_{z}(z) & =\int_{-\infty}^{\infty} P_{z_{1} z_{2}}\left(z_{1}, z-z_{1}\right) d z_{1} \\
& =\int_{-\infty}^{\infty} P_{z_{1}}\left(z_{1}\right) P_{z_{2}}\left(z-z_{1}\right) d z_{1} \text { (uncorrelated) }
\end{aligned}
$$

To compute the PDF of $\widehat{Z}$, we just continue the process

$$
\widehat{z}_{123}=\widehat{z}_{12}++\widehat{z}_{3},
$$

so

$$
\widehat{Z}=\widehat{z}_{123 \ldots N-1}+\widehat{z}_{N} .
$$

In principle the problem is solved provided the PDF for $f_{z}(z)$ can be calculated so one can then calculate $P_{z}(z)$. Calculations of convolution of Chebyshev polynomials are not difficult, so we can calculate,all of these sums.

Note if one is solely concerned with the first and second moments of the distributions, the individual distributions can be used to compute the collective means and standard deviations for $\widehat{Z}$. For the Gaussian distribution, the mean is zero and the second moment is (where we have replaced $R$ with the amplitude $A$ )

$$
\left\langle x^{2}\right\rangle=2 A^{2}\left(1-e^{-2 \sigma_{\theta}^{2}}\right)
$$

Thus for case 1 we have

$$
\left\langle Z^{2}\right\rangle=2 N A^{2}\left(1-e^{-2 \sigma_{\theta}^{2}}\right)
$$

for case 2 we have

$$
\left\langle Z^{2}\right\rangle=2 N A^{2}-2 A^{2} \sum_{i=1}^{N} e^{-2 \sigma_{\theta_{i}}^{2}},
$$

for case 3 we have

$$
\left\langle Z^{2}\right\rangle=2\left(1-e^{-2 \sigma_{\theta}^{2}}\right) \sum_{i=1}^{N} A_{i}^{2},
$$

and for case 4 we have

$$
\left\langle Z^{2}\right\rangle=2 \sum_{i=1}^{N} A_{i}^{2}\left(1-e^{-2 \sigma_{\theta_{i}}^{2}}\right) .
$$

Note when the mean is not zero, we have

$$
\langle Z\rangle=2 e^{-\sigma_{\theta}^{2} / 2}\left[\cos \theta_{0}\right] A,
$$

and the second moment is

$$
\left\langle Z^{2}\right\rangle=2\left[1-e^{-2 \sigma_{\theta}^{2}} \cos \left(2 \theta_{0}\right)\right] A^{2} .
$$

and we replace the previous cases with $e^{-2 \sigma_{\theta}^{2}} \cos \left(2 \theta_{0}\right)$ for $e^{-2 \sigma_{\theta}^{2}}$ with the proper index as necessary. State of the art signal processing requires using statistics beyond second order so that requires returning to PDF's of the scatters and evaluating their convolutions.

Now we know the functional form for the sinusoidal transform is either (43) or (44). For the last two cases we need the formula for the density function for the product of two random variables $\widehat{z}=\widehat{s} \widehat{t}$ which is given by

$$
f_{z}(z)=\int_{-\infty}^{\infty} \frac{1}{|w|} f_{s t}\left(w, \frac{z}{w}\right) d w \equiv \int_{-\infty}^{\infty} \frac{1}{|w|} f_{s}(w) f_{t}\left(\frac{z}{w}\right) d w,
$$

when $s$ and $t$ are independent. (Note, by symmetry, it doesn't matter which order of labeling, $s$ or $t$ one chooses in the integral.) It does matter whether we use sine or cosine since once one integral is evaluated the other can be evaluated in terms of the first, so the cosine is considered in the remainder of the paper. The probability density function for the transformation $z=\hat{a} \cos (\hat{\theta})$ is $\left(f_{a}(w)\right.$ is the PDF for $\left.\hat{a}\right)$

$$
f_{z}(z)=\frac{2}{\pi} \int_{-1}^{1} \frac{\left[F(0)+\sum_{n=1}^{\infty} U_{\cos (\theta)}(n) T_{n}(w)\right]}{|w| \sqrt{1-w^{2}}} f_{a}\left(\frac{z}{w}\right) d w .
$$

There are two limiting cases for $f_{a}(w)$ that are of particular interest, a uniformly distributed amplitude

$$
f_{a}(w)=\frac{1}{2 a} \Theta\left(1-\left|\frac{w}{a}\right|\right)
$$

and a normally distributed amplitude

$$
f_{a}(w)=\frac{1}{\sqrt{2 \pi \sigma_{a}^{2}}} \exp \left(-\frac{w^{2}}{2 \sigma_{a}^{2}}\right) .
$$

For the uniformly distributed amplitude

$$
\begin{aligned}
f_{z}(z)= & \frac{F(0)}{\pi a} \int_{-1}^{1} \frac{\Theta\left(1-\left|\frac{z}{a w}\right|\right)}{|w| \sqrt{1-w^{2}}} d w \\
& +. \frac{\sum_{n=1}^{\infty} U_{\cos (\theta)}(n)}{a \pi} \int_{-1}^{1} \frac{T_{n}(w) \Theta\left(1-\left|\frac{z}{a w}\right|\right)}{|w| \sqrt{1-w^{2}}}(\text { (60) })
\end{aligned}
$$

Now the first integral is even, and it can be evaluated in closed form [13] 341.01

$$
\int \frac{1}{|x| \sqrt{b^{2}-x^{2}}} d x=-\frac{1}{b} \log \left|\frac{b+\sqrt{b^{2}-x^{2}}}{x}\right| .
$$

First note that $T_{n}(-w)=(-)^{n} T_{n}(w)$, and [14]

$$
T_{n}(x)=\sum_{k=0}^{[n / 2]} c_{k}^{(n)} x^{n-2 k}
$$


where

$$
c_{k}^{(n)}=(-)^{k} 2^{n-2 k-1} \frac{n}{n-k}\left(\begin{array}{c}
n-k \\
k
\end{array}\right) .
$$

Now the definition of the beta function is

$$
B(m, n)=\int_{0}^{1} x^{m-1}(1-x)^{n-1} d x
$$

while definition of the gamma function is

$$
\Gamma(m+1)=\int_{0}^{\infty} x^{m} e^{-x} d x,
$$

so

$$
B(m, n)=\frac{\Gamma(m) \Gamma(n)}{\Gamma(m+n)} .
$$

The definition of the incomplete beta function is

$$
B_{u}(a, b)=\int_{0}^{u} x^{a-1}(1-x)^{b-1} d x \operatorname{Re}(a)>0
$$

while the complementary incomplete beta function is

$$
B_{u}^{c}(a, b)=\int_{u}^{1} x^{a-1}(1-x)^{b-1} d x \operatorname{Re}(a)>0
$$

so

$$
B_{u}(a, b)+B_{u}^{c}(a, b)=B(a, b) .
$$

Then the integral is expressible in terms of the complementary incomplete beta function as

$$
\int_{\frac{a}{z}}^{1} \frac{w^{2 m-2 k-1}}{\sqrt{1-w^{2}}} d w=B_{\frac{a}{z}}^{c}\left(m-k-1, \frac{1}{2}\right) .
$$

The PDF for an arbitrary phase noise and uniform amplitude noise follows from these integrals and is

$$
\begin{aligned}
f_{z}(z)= & \frac{F(0)}{\pi a} \log \left|\frac{z+\sqrt{z^{2}-a^{2}}}{a}\right|+ \\
& \frac{\sum_{m=1}^{\infty} U_{\cos (\theta)}(2 m) \sum_{k=0}^{[m]} c_{k}^{(2 m)} B_{\frac{a}{z}}^{c}\left(m-k-1, \frac{1}{2}\right)}{a \pi}
\end{aligned}
$$

For the normally distributed amplitude, the PDF is

$$
\begin{aligned}
f_{z}(z)= & \frac{2 F(0)}{\pi \sqrt{2 \pi \sigma_{a}^{2}}} \int_{0}^{1} \frac{\exp \left(-\frac{z^{2}}{2 w^{2} \sigma_{a}^{2}}\right)}{w \sqrt{1-w^{2}}} d w+ \\
& \frac{\sum_{n=1}^{\infty} U_{\cos (\theta)}(n)}{\pi \sqrt{2 \pi \sigma_{a}^{2}}} \int_{-1}^{1} \frac{T_{n}(w) \exp \left(-\frac{z^{2}}{2 w^{2} \sigma_{a}^{2}}\right)}{|w| \sqrt{1-w^{2}}} \text { (74) }
\end{aligned}
$$

The definition of the incomplete gamma function is

$$
\Gamma_{z}(m+1)=\int_{0}^{z} x^{m} e^{-x} d x,
$$

while the definition of the complementary incomplete gamma function is

$$
\Gamma_{z}^{c}(m+1)=\int_{z}^{\infty} x^{m} e^{-x} d x
$$

so

$$
\Gamma_{z}(m+1)+\Gamma_{z}^{c}(m+1)=\Gamma(m+1)
$$

Now the first integral in the PDF can be evaluated as $(k(z)=$ $\left.\frac{z^{2}}{2 \sigma_{a}^{2}}\right)$

$$
\begin{aligned}
& \int_{0}^{1} \frac{\exp \left(-\frac{z^{2}}{2 w^{2} \sigma_{a}^{2}}\right)}{w \sqrt{1-w^{2}}} d w \\
= & \frac{1}{2} \sum_{n=0}^{\infty}(-)^{n} \frac{(2 n-1) ! ! \Gamma_{k(z)}^{c}\left(n+\frac{1}{2}\right)}{(2 n) ! ! k(z)^{n+\frac{1}{2}}} \\
= & \frac{1}{2} \sum_{n=0}^{\infty}(-)^{n} \frac{(2 n-1) ! !}{(2 n) ! !} R(n, k(z))
\end{aligned}
$$

The second integral can be expressed as

$$
\begin{aligned}
& \int_{-1}^{1} \frac{T_{n}(w) \exp \left(-\frac{z^{2}}{2 w^{2} \sigma_{a}^{2}}\right) d w}{|w| \sqrt{1-w^{2}}} \\
= & \frac{1}{2} \sum_{m=1}^{\infty} \frac{(4 m-1) ! ! H(m, k(z))}{(4 m) ! ! k(z)^{2 m+\frac{1}{2}}}
\end{aligned}
$$

where

$$
H(m, k(z))=\sum_{l=0}^{[m]} \frac{c_{l}^{(2 m)}}{k(z)^{l}} \Gamma_{k(z)}^{c}\left(l+n-m+\frac{1}{2}\right)
$$

since it is even. Thus, PDF for an arbitrary phase noise and Gaussian amplitude noise follows from these integrals and is given by

$$
\begin{aligned}
f_{z}(z)= & \frac{F(0)}{\pi \sqrt{2 \pi \sigma_{a}^{2}}} \sum_{n=0}^{\infty}(-)^{n} \frac{(2 n-1) ! !}{(2 n) ! !} R(n, k(z)) \\
& +. \frac{\sum_{m=1}^{\infty} U_{\cos (\theta)}(2 m) \frac{(4 m-1) ! ! H(m, k(z))}{(4 m) ! ! k(z)^{2 m+\frac{1}{2}}}}{2 \pi \sqrt{2 \pi \sigma_{a}^{2}}}
\end{aligned}
$$

We have shown it is possible to determine the PDF for specific models of amplitude noise and arbitrary phase noise. From these expressions, one can work out either the moments or (72) the characteristic function of the distributions. Once the PDF has been determined for $\hat{z}$, sums can be considered. Let us consider the two sum case for each of these possibilities and see what conclusions we can draw. For the two sum case $\widehat{Z}=$ $\widehat{z}_{1}+\widehat{z}_{2}$, we know the PDF of $\widehat{Z}$ is

$$
P_{Z}(z) \stackrel{\text { (uncorrelated) }}{=} \int_{-\infty}^{\infty} P_{z_{1}}\left(z_{1}\right) P_{z_{2}}\left(z_{1}-z\right) d z_{1} .
$$

Thus our ability to determine $P_{Z}(z)$ depends on our ability to evaluate for either sine or cosine integrals of the form so our anzatz for the general case applies by computing the sums two-fold at a time. Details of these calculations and explicit determination of the moments will be considered in a future paper.

\section{BIOGRAPHY FORMAT}

John E. Gray

Naval Surface Warfare Center Dahlgren Division

Code B-10

Dahlgren, VA 22448

john.e.gray@navy.mil 


\section{REFERENCES}

[1] D. C. Schleher, MTI and Pulsed Doppler Radar, Artech House Publishers, 1991.

[2] J. B. Billingsley, Low Angle Land Clutter Measurements and Empirical Models, William Andrew Publishing, 2002

[3] Lord Rayleigh, "On the Problem of Random Vibrations and of Random Flights in One, Two and Three Dimensions," Philos. Mag., 37, 321-347, 1919.

[4] P. Beckmann and A. Spizzichino, The Scattering of Electromagnetic Waves from Rough Surfaces, Artech House Inc., 1987.

[5] R. Barakat, "Probability Density Functions of Sums of Sinusoidal Waves Having Non uniform Random Phases and Random Numbers of Multipath", J. Acoust. Soc Am., 83 (3) March 1988.

[6] E. Lukacs, Characteristic Functions, Hafner Publishing Company, New York, 1960.

[7] A. Papoulis, Probability, Random Variables, and Stochastic Processes, Third Edition, McGraw-Hill Book Company, 1991.

[8] G. Arfken, Mathematical Methods for Physicists, Academic Press, Third Edition, 1985.

[9] L. Cohen, "Time-Frequency Analysis", Prentice-Hall, 1994.

[10] J. E. Gray and S. R. Addison, "Characteristic Functions in Radar and Sonar", Thirty-Fourth Southeastern Symposium on System Theory March 18, 2002.

[11] J. E. Gray and S. R. Addison, "A methodology for characterizing phase noise in radar waveforms: an alternative "Terrain" characterization method", Proceedings of SPIE, Vol. 5410: Radar Sensor Technology VIII and Passive Millimeter-Wave Imaging Technology VII, April 15, 2004.

[12] M. Abramowitz and I. A. Stegun, Handbook of Mathematical Functions, National Bureau of Standards Applied Mathematics Series 55, Fourth Printing, December, 1965.

[13] H. B. Dwight, Tales of Integrals and Other Mathematical Data, Fourht Edittion, MacMillian, 1961

[14] J. C. Mason and D. C. Handscomb, Chebyshev Polynomials, Chapman \& Hall/CRC, 2003

[15] I. H. Sneddon, The Use of Integral Transforms, McGraw-Hill, 1972.

\section{BIOGRAPHY}

John E. Gray received Bachelor of Science degrees in mathematics and physics from the University of Mississippi in Dec. 1977. He received a Masters Degree in physics from he University of Mississippi in August 1980. Additionally he has taken electrical engineering courses from the Catholic University and physics courses from VPI.

He has worked at the Naval Surface Warfare Center over twenty-one years. He has worked in the areas of radar, electromagnetic, signal processing, track filtering, weapon's control systems, and guidance algorithms. He has over one hundred technical publications in these areas as well as physics. He has served on the publication and scientific committee for several national and international conferences. He has served as a referred for IEEE journals and Radio Science. He has been invited to present papers at both national and international conferences.

$\mathrm{He}$ is a member of the IEEE, Society for Industrial and Applied Mathematics (SIAM), Life Member of the American Physical Society (APS), SPIE, Sigma Phi Sigma, American Association for the Advancement of Sciences (AAAS), and the Washington Evolutionary Systems Society (WESS). 